\title{
Bilateral variation of the venous corona mortis with a presentation previously undescribed
}

\author{
J. Pinochet, C.R. Molina, E.Y. Flores \\ Department of Anatomy, Faculty of Medicine, Finis Terrae University, Santiago, Chile \\ [Received: 2 June 2015; Accepted: 3 September 2015]
}

\begin{abstract}
The corona mortis is an anatomic variant that involves anastomosis between obturator vessels, external iliac vessels and/or inferior epigastric vessels. It is clinically and surgically important because its section may lead to fatal consequences when pelvic procedures are performed. We report a case in which, during an anatomical dissection in a female cadaver, a bilateral corona mortis that involved obturator vessels and external iliac vessels was found, presenting differences on each side, an undescribed type of corona mortis was found and a classification modification was proposed. (Folia Morphologica 2016; 74, 3: 409-412)
\end{abstract}

Key words: corona mortis, obturator vessels, external iliac vessels

\section{INTRODUCTION}

The corona mortis (CMOR) or "corona of death" is an anatomical variant, in which an anastomosis occurs between the obturator vessels and the external iliac or inferior epigastric arteries or veins $[9,11,13]$. Its name derives from the heavy bleeding generated on being sectioned. This may cause death due to a difficult haemostasis or may go unnoticed $[2,11,15]$.

Darmanis et al. [2] described a paradox of this variation: Although, in anatomic dissections, a corona mortis is considered a relevant vessel, in clinical practice it would be considered a small-sized vessel [11]. However, multiple authors describe the relevance of the risk of bleeding that exists when the variation is ignored, and various other authors agree on the clinical and surgical relevance of CMOR trauma and its importance in the performance of laparoscopic surgical procedures: for example, hernia resolution, handling the spermatic cord etc. [1, 7, 14]. Episodes of heavy bleeding and even deaths associated with the ignorance of this variant have been described.

This anastomosis is related to the anterior pectineal ligament, which stands at a variable distance from the symphysis pubis. Nevertheless, it crosses over the pecten pubis. The CMOR may have a venous or arterial origin; however, there are cases where both coexist. Of these origins, the first one is the most common, followed by the arterial and venous origin, with the arterial origin being the least common [1]. The incidents described have a wide variability, showing $20-80 \%$ for venous and 14-28\% for arterial anastomosis [1, 2, 4-6, 8-15]. Apparently, there is no significant difference in the incidence between men and women, nor in the distance between the involved vessels and the symphysis pubis [11].

A case of cadaver dissection is presented next in which the existence of a bilateral CMOR with an anatomical variant unclassified in the literature was identified.

\section{CASE REPORT}

The case is a cadaver of female sex, Caucasian, 81 years old, with no history of pelvic or abdominal hernia or surgical procedure in the infraumbilical region. The abdominal wall was dissected by removing both rectus abdominis muscles, exposing the inguinal canal and the pecten pubis bilaterally. The findings are described below.

\section{Right side}

There are four structures observed in the obturator foramen: a vein support for the obturator vein (OV), 


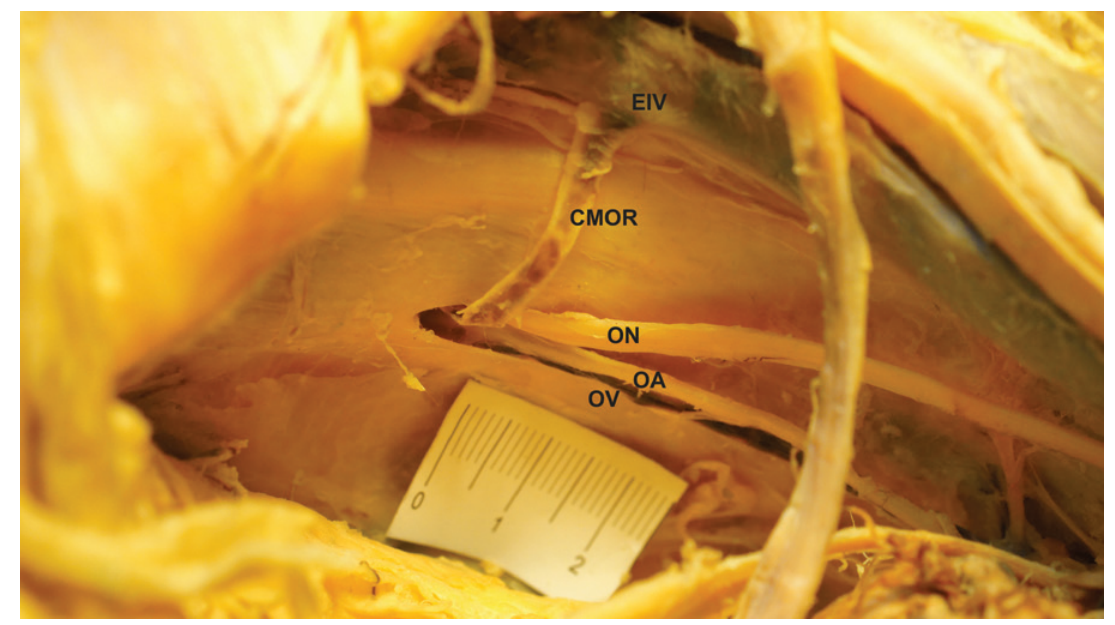

Figure 1. Right side of corona mortis (CMOR) and its relationship with the obturator and external iliac systems; EIV — external iliac vein; $\mathrm{ON}$ - obturator nerve; $\mathrm{OA}$ - obturator artery; $\mathrm{OV}$ — obturator vein.

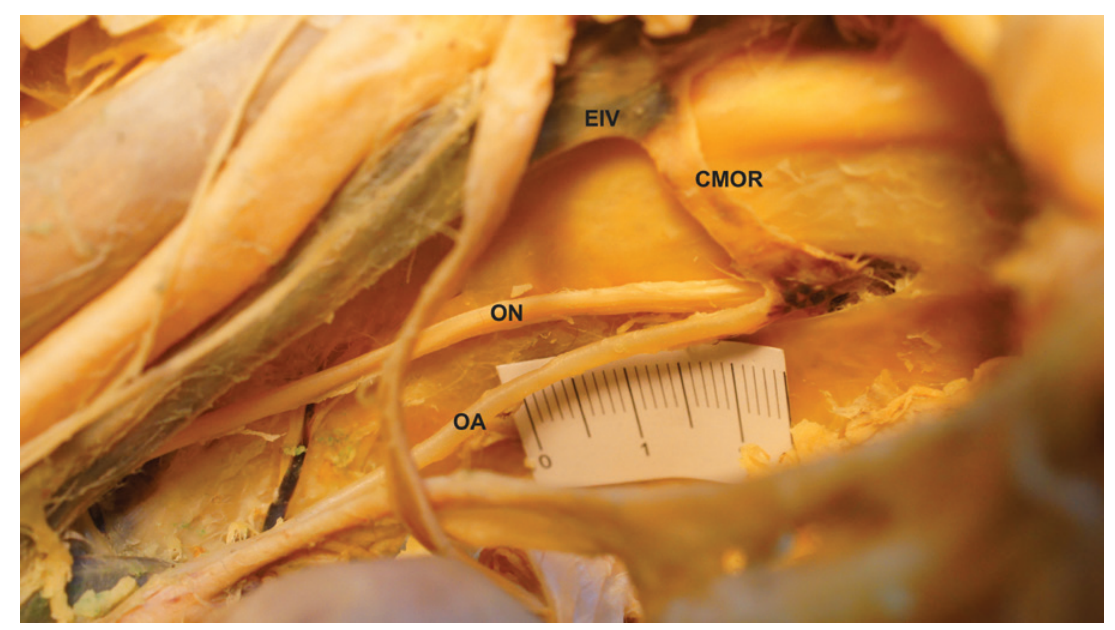

Figure 2. Left side of corona mortis (CMOR) and its relationship with the obturator and external iliac systems; EIV — external iliac vein; $\mathrm{ON}$ - obturator nerve; $\mathrm{OA}$ - obturator artery.

and above this, the obturator artery (OA). Above these two is located the obturator nerve (ON). Immediately from where the OV emerges through the obturator duct, there is an anastomosis compatible with CMOR following a slightly inclined position on the vertical axis. From anterior to posterior it passes over the superior branch of the pubis and flows into the external iliac vein (EIV) (Fig. 1). This CMOR measures $33 \mathrm{~mm}$ in length on the path described, and has a gauge of $3 \mathrm{~mm}$. The distance from the pubic symphysis to the mouth of the EIV is $67 \mathrm{~mm}$, while the distance from the junction of the CMOR and EIV to the lacunar ligament is $19 \mathrm{~mm}$. The distance from the pubic symphysis to the other end of the CMOR in the obturator foramen is $53 \mathrm{~mm}$.

\section{Left side}

Three structures are observed in the obturator foramen: the OV, the OA, and above this, the ON. Below the artery, there is a vein, emerging from the obturator foramen, compatible with the CMOR (Fig. 2). It has a length of $18 \mathrm{~mm}$ on the path described and an irregular gauge of $8 \mathrm{~mm}$. The distance from the pubic symphysis to the place of arrival of the CMOR in the EIV is $65 \mathrm{~mm}$. The distance from the binding of the CMOR and the EIV to the lacunar ligament is $15 \mathrm{~mm}$. The distance from the pubic symphysis to the other end of the CMOR in the obturator foramen is $60 \mathrm{~mm}$.

It should be mentioned that it is not possible to identify an $\mathrm{OV}$ in the obturator foramen. 


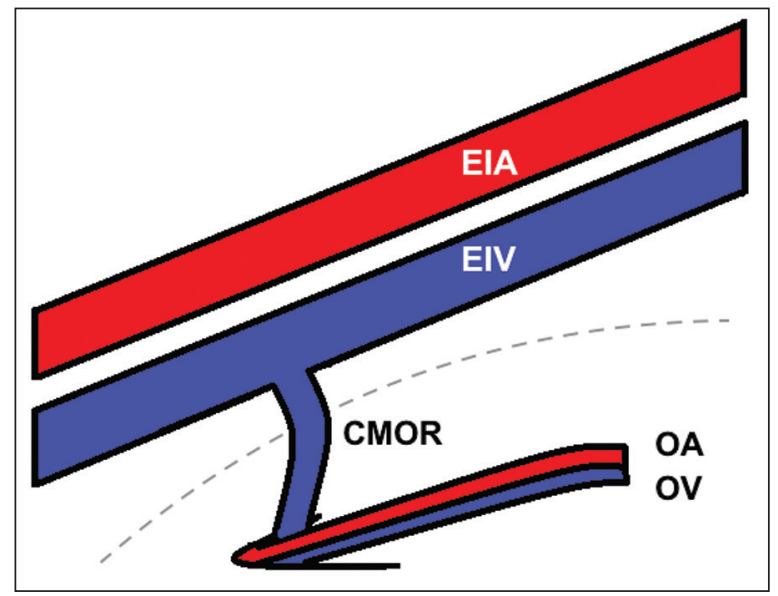

Figure 3. Diagram of the new type of corona mortis (CMOR); EIA — external iliac artery; EIV — external iliac vein; OV — obturator vein; $\mathrm{OA}$ - obturator artery.

\section{DISCUSSION}

There are several papers describing cases of CMOR and identifying differences in the laterality. However, those papers do not describe whether the CMOR has similar features on both sides, or whether it presents variations on the contralateral side in the same individual, as was observed in the case described above.

According to the classification proposed by Rusu et al. [11], the case presented in this paper has a vein compatible with a CMOR type (II2) on the left side. However, the CMOR on the right side does not agree fully with any classification. Although it resembles the type (II3), it follows a different route.

Therefore, we suggest changing the classification described by Rusu et al. [11], changing the type (II3) to (II3a), and adding the new type (II3b) following the pattern of CMOR described on the right side of the case presented, with this classification being in accordance with Figure 3.

The significance of this variation is understood, in light of medical procedures in which an increased risk of bleeding associated with CMOR has been seen. Among these procedures, in gynaecology, transobturator vaginal tape has proven to be quite safe with low risk in connection with this variation. They have also found that with the proper technique and following the medial border of the obturator foramen, in most cases there would be a window of at least $8 \mathrm{~mm}$ between the margin of the transobturator vaginal tape and most of the aberrant veins. In the case of arteries, this distance is even greater [13].
This anatomical variation is probably more relevant in traumatology, involving fractures of the pubis, where not only may the fracture act as a risk factor for bleeding, but the osteosynthesis material located in this region must also take this variation into consideration $[3,6,7]$. The same is true for gastric surgeons involved in inguinal hernias, which may be located precisely in this variation, increasing the risk of bleeding [9].

While some authors have shown that CMOR can be seen by computed tomography, in the absence of a fracture, using this image to identify the presence of the vascular alteration mentioned is questionable [14]. We believe that the decision must be made on a case-by-case basis, taking the radiation to which the individual is subjected to into consideration along with the potential risk of bleeding.

\section{CONCLUSIONS}

The unexpected presence of vascular variants in the retropubic region can be of great importance for physicians who perform surgical operations in the region. A type not previously described in the classifications of CMOR that must be identified in order to intervene in the retropubic region is presented in this research. The case illustrated also confirms that the presence of CMOR may differ in relation to its contralateral side, not only in distance. It also differs in size and length.

\section{REFERENCES}

1. Berberoğlu M, Uz A, Ozmen MM, Bozkurt MC, Erkuran C, Taner S, Tekin A, Tekdemir I (2001) Corona mortis: an anatomic study in seven cadavers and an endoscopic study in 28 patients. Surg Endosc, 15: 72-75.

2. Darmanis S, Lewis A, Mansoor A, Bircher M (2007) Corona mortis: an anatomical study with clinical implications in approaches to the pelvis and acetabulum. Clin Anat $\mathrm{N}$ Y N, 20: 433-439.

3. Garrido-Gómez J, Pena-Rodríguez C, Martín-Noguerol T, Hernández-Cortes P (2012) Corona mortis artery avulsion due to a stable pubic ramus fracture. Orthopedics, 35 : e80-e82.

4. Gobrecht U, Kuhn A, Fellman B (2011) Injury of the corona mortis during vaginal tape insertion (TVT-SecurTM using the U-Approach). Int Urogynecology J, 22: 443-445.

5. Hong H, Pan Z, Chen X, Huang Z (2004) An anatomical study of corona mortis and its clinical significance. Chin J Traumatol Zhonghua Chuang Shang Za Zhi Chin Med Assoc, 7: 165-169.

6. Karakurt L, Karaca I, Yilmaz E, Burma O, Serin E (2002) Corona mortis: incidence and location. Arch Orthop Trauma Surg, 122: 163-164. 
7. Lau H, Lee F (2003) A prospective endoscopic study of retropubic vascular anatomy in 121 patients undergoing endoscopic extraperitoneal inguinal hernioplasty. Surg Endosc, 17: 1376-1379.

8. Okcu G, Erkan S, Yercan HS, Ozic U (2004) The incidence and location of corona mortis: a study on 75 cadavers. Acta Orthop Scand, 75: 53-55.

9. Pellegrino A, Damiani GR, Marco S, Ciro S, Cofelice V, Rosati $F$ (2014) Corona mortis exposition during laparoscopic procedure for gynecological malignancies. Updat Surg, 66: 65-68.

10. Pungpapong S, Thum-umnauysuk S (2005) Incidence of corona mortis: preperitoneal anatomy for laparoscopic hernia repair. J Med Assoc Thail Chotmaihet Thangphaet, 88 (suppl. 4): S51-S53.
11. Rusu MC, Cergan R, Motoc AGM, Folescu R, Pop E (2010) Anatomical considerations on the corona mortis. Surg Radiol Anat SRA, 32: 17-24.

12. Sarikcioglu L, Sindel M, Akyildiz F, Gur S (2003) Anastomotic vessels in the retropubic region: corona mortis. Folia Morphol, 62: 179-182.

13. Stavropoulou-Deli A, Anagnostopoulou (2013) S. Corona mortis: anatomical data and clinical considerations. Aust N Z J Obstet Gynaecol, 53: 283-286.

14. Smith JC, Gregorius JC, Breazeale BH, Watkins GE (2009) The corona mortis, a frequent vascular variant susceptible to blunt pelvic trauma: identification at routine multidetector CT. J Vasc Interv Radiol, 20: 455-460.

15. Tornetta P, Hochwald N, Levine R (1996) Corona mortis. Incidence and location. Clin Orthop, 329: 97-101. 